\title{
Should molecular subtype be recommended as one of the selection criteria for accelerated partial breast irradiation? Preliminary results from an Asian cohort
}

\author{
Tabassum Wadasadawala, MD, DNB', Monidipa Mondal, MD², Siji Nojin Paul, DipRPP3, Vani Parmar, MS, \\ Nita Nair, MS ${ }^{5}$, Tanuja Shet, MD6, Sangeeta Desai, MD', Sudeep Gupta, MD, DM7, Rajiv Sarin, MD, FRCR ${ }^{2}$ \\ 'Department of Radiation Oncology, Advanced Centre for Treatment, Research \& Education in Cancer (ACTREC), Tata Memorial Centre (TMC). \\ Kharghar, Navi Mumbai, ${ }^{2}$ Department of Radiation Oncology, Tata Memorial Hospital (TMH), Parel, Mumbai, ${ }^{3}$ Department of Radiation \\ Oncology (Medical Physics), ACTREC, TMC, Kharghar, Navi Mumbai, ${ }^{4}$ Department of Surgical Oncology, ACTREC, TMC, Kharghar, Navi \\ Mumbai, ${ }^{5}$ Department of Surgical Oncology, TMH, Parel, Mumbai, ${ }^{6}$ Department of Pathology, TMH, Parel, Mumbai, ${ }^{7}$ Department of Medical \\ Oncology, ACTREC,TMC, Kharghar, Navi Mumbai, India
}

\begin{abstract}
Purpose: The purpose of this study was to report clinical outcomes in patients treated with accelerated partial breast irradiation (APBI), stratified as per molecular subtype and American Society for Therapeutic Radiology and Oncology/ Groupe Européen de Curiethérapie and European Society for Radiotherapy \& Oncology (ASTRO/GEC-ESTRO) patient selection criteria in order to determine whether molecular subtype should be recommended as one of the selection criteria for APBI.

Material and methods: 157 early-stage breast cancers patients, treated with APBI using multi-catheter interstitial brachytherapy with $\geq 6$ months follow-up were included. Molecular subtype was assigned based on estrogen/progesterone receptor (ER/PR), Her2neu and tumor grade. Patients were stratified into ASTRO and GEC-ESTRO risk groups, as per updated ASTRO consensus statement (CS) and GEC-ESTRO recommendation, respectively. The Kaplan-Meier method was used to calculate the time to event data of clinical outcomes.

Results: With a median follow-up of 35 months, local control (LC) and locoregional control (LRC) were not significantly different among the different molecular subtypes $(p=0.19, p=0.41$, respectively). None of the APBI guidelines predicted risk of local or locoregional recurrence. Re-analyzing the data by replacing ER status with molecular subtype in the ASTRO-CS did not show any significant difference in LC/LRC across the various categories. Her2neu subtype was associated with significantly lower disease-free survival, cause specific survival, and overall survival than the luminal subtypes.

Conclusions: None of the mentioned APBI guidelines predicted local or locoregional recurrence risk in our study population. Additional follow-up will be needed to recommend inclusion of molecular subtype (or at least HER2 receptor status) in the patient selection criteria for APBI.

Key words: accelerated partial breast irradiation, breast cancer, multicatheter interstitial brachytherapy.

\section{Purpose}

Breast conserving surgery (BCS) and radiotherapy (RT) to the conserved breast is considered as a standard treatment in early stage breast cancer. Accelerated partial breast irradiation (APBI) is a form of hypo-fractionated regimen, in which only the lumpectomy bed plus a $1-2 \mathrm{~cm}$ margin is treated, rather than conventional whole breast irradiation (WBI). It is an appealing alternative to WBI for selected group of patients with favorable early-stage disease because of shortened treatment time with reduced radiation exposure to the surrounding normal tissues, which include the uninvolved breast, skin, heart, and lungs. A recently published randomized phase 3 study by the Groupe Européen de Curiethérapie and the European Society for Radiotherapy \& Oncology (GEC-ESTRO) showed non-inferiority of adjuvant APBI using multicatheter interstitial brachytherapy (MIB-APBI) to adjuvant WBI after BCS in early breast cancer. The 5-year local control, disease-free survival, and overall survival were similar in the two arms, 
along with lesser rate of late normal tissue toxicity with APBI [1]. Cost simulation based on the result of this study has shown that APBI is a cost-effective alternative to WBI [2]. Satisfaction with the cosmetic outcome and quality of life scores are also higher with MIB-APBI compared to WBI [3].

Patient selection has an important implication on the outcomes of APBI. Although, several expert consensus groups like the GEC-ESTRO, American Society for Therapeutic Radiology and Oncology (ASTRO), American Brachytherapy Society (ABS), American Society of Breast Surgeons (ASBS), National Surgical Adjuvant Breast and Bowel Project (NSABP), and Radiation Therapy Oncology Group (RTOG) have developed guidelines for selection of patients for APBI, there is lack of consistency among the recommendations and clinical applicability of these guidelines $[4,5,6,7,8,9]$. The ASBS and ABS guidelines do not comment on important prognostic factor like multifocality or multicentricity. Moreover, all these guidelines are based mainly on histopathological factors and do not incorporate molecular subtypes of breast cancer. ASTRO guidelines have included only estrogen receptor (ER) status but other consensus guidelines like GEC-ESTRO, ABS, and ASBS have not included receptor status or grade in their recommendations [4]. Patient selection criteria according to various consensus guidelines are summarized in Table 1. There is ample literature on the impact of molecular subtype on distant recurrence and overall survival, which is used routinely to guide systemic therapy treatment decisions. There is also growing evidence that molecular phenotype has prognostic significance for locoregional recurrence risk both following APBI as well as WBI $[10,11,12,13,14,15,16,17,18]$.

Hence, we conducted a study with an aim to report early clinical outcome in patients treated with MIB-APBI in our institution, stratified as per molecular subtype and ASTRO/GEC-ESTRO patient selection criteria.

\section{Material and methods \\ Study participants and treatment method}

All consecutive women $(n=157)$ with early stage invasive breast cancers, who underwent BCS and either intra operative or post-operative high-dose-rate (HDR) MIB-APBI from January 2012 to July 2016, and had at least 6 months of follow-up were included in the current analysis. Patient demographics including age, stage, tumor characteristics and treatment details, toxicity and clinical outcome data were recorded, compiled, and analyzed.

For patients who underwent intra-operative APBI, histopathology was reviewed with frozen section after lumpectomy and axillary sampling to confirm that the tumor size, margin, and nodal status are as per the APBI eligibility criteria, which have been reported earlier [19]. The implant procedure, planning, implant quality, and dosimetric indices have also been described in this report. Patients who had undergone BCS using open cavity technique were considered for APBI. Patients underwent brachytherapy either in intra-operative or post-operative setting, depending on the time of referral to our institute.
If the patient was referred pre-operatively, the patient was taken up for BCS with intra-operative implant in the same setting. In the setting of patients who had already undergone excision biopsy or BCS, suitable patients were considered for post-operative implant, which was done as a second procedure. In both the cases, APBI was delivered using multicatheter interstitial HDR brachytherapy. Result from the previous study showed that there were no significant differences in the dosimetric parameters, based on whether the technique was done intra-operatively or post-operatively [19]. Planning was done on Oncentra planning system (version 4.3; Nucletron, Elekta, Stockholm, Sweden), and a dose fractionation of 32-34 Gy, in 8-10 twice daily fractions, over 5-7 days was used. During 2012-2015, 34 Gy/10 fractions/5 days was used in accordance with the ABS guidelines, while $32 \mathrm{~Gy} /$ 8 fractions/ 4 days was used from 2016 onwards, following published results of the GEC-ESTRO trial. Adjuvant chemotherapy and/or hormonal therapy were given as per institutional guidelines. Molecular subtype was assigned based on immune-histochemistry (IHC) for the estrogen-progesterone receptors and Her2neu status. Tumor grade was used as a surrogate measure of proliferation (Table 2). All patients were stratified into ASTRO groups (suitable, cautionary, and unsuitable) as per updated ASTRO consensus statement, and ESTRO risk categories (low, intermediate, high-risk) as per GEC-ESTRO recommendation [5,9].

Patients were followed up every 6 months after completion of radiotherapy for 5 years. Clinical examination was performed on each follow-up visit and bilateral mammography was obtained once in every 18 months. Those patients who were unable to come for a follow-up in last 6 months were called telephonically to know their disease status.

\section{Endpoints and statistical analysis}

Statistical analysis was performed using Statistical Programme for Social Sciences software for Windows (SPSS Inc., Version 20). Patient, tumor, and treatment characteristics were reported as numbers and proportions. Tumor and treatment characteristics across the subgroups were compared using Pearson Chi-square test. $P$ value $<0.05$ was considered to be significant. Normality of the quantitative variables was tested using Shapiro-Wilk test. Clinical outcomes that were studied included local control (LC), locoregional control (LRC), distant metastasis free survival (DMFS), disease-free survival (DFS), cause specific survival (CSS), and overall survival (OS). All the time to event data were calculated from the date of surgery. Kaplan-Meier curves were used to generate various time to event curves, and log-rank statistical test was applied to determine the difference between actuarial rates. Variables were also compared pairwise over strata to find out potential meaningful difference in outcome. Univariate and multivariate (using Cox proportional hazard model) analyses was performed to estimate the risks of LRC and DFS, using the following variables: molecular subtype, updated ASTRO group, GEC-ESTRO category, age group, grade, pathological tumor size, 


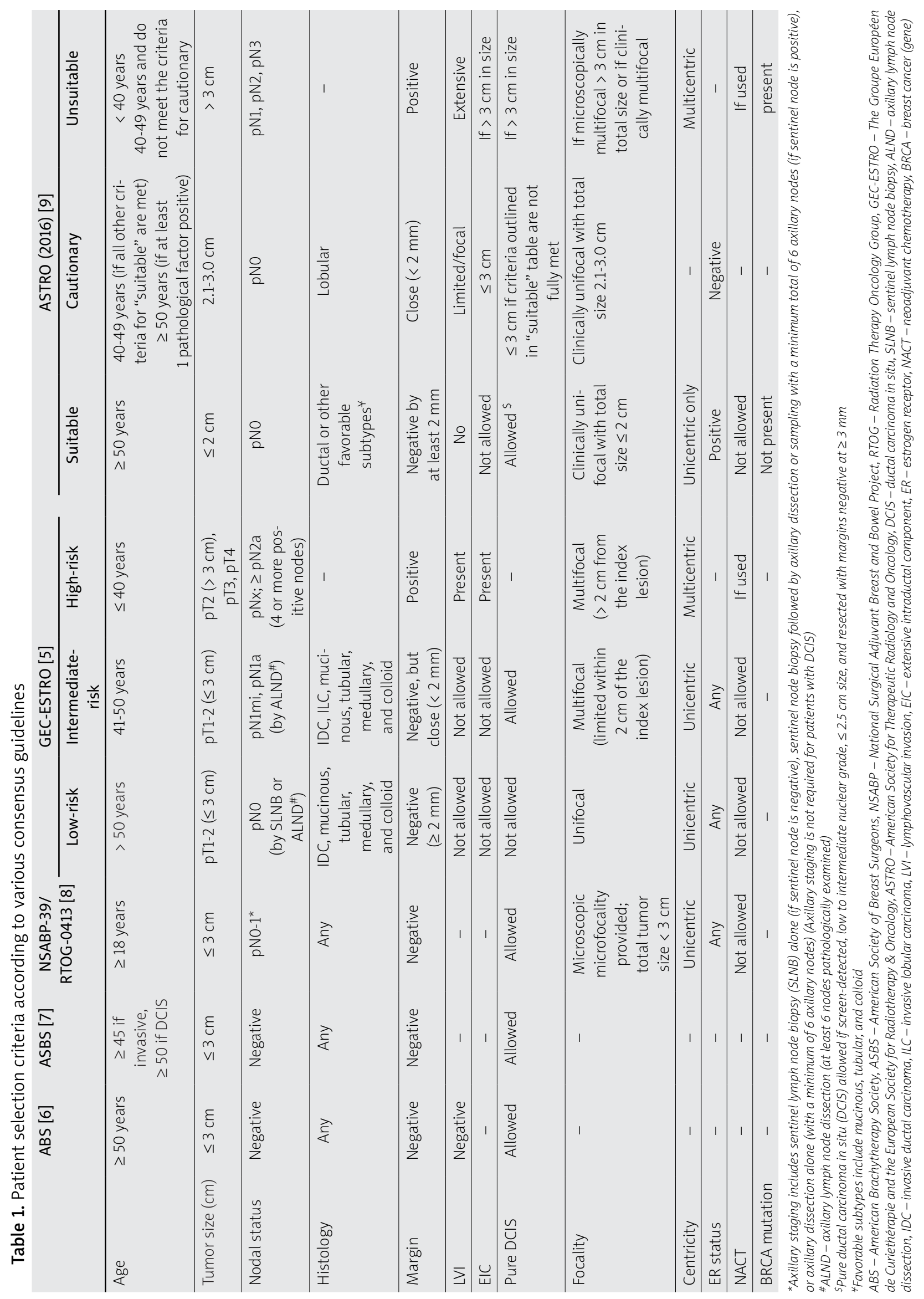


Table 2. Molecular subtypes: breast cancer molecular subtype assignment was done based on immunehistochemistry (IHC) for the estrogen-progesterone receptors and Her2neu status. Tumor grade was used as a surrogate measure of proliferation

\begin{tabular}{lc} 
Molecular subtype & Receptor status \\
\hline Luminal A & ER+ and/or PR+, HER2- and grade I or II \\
\hline Luminal B & $\begin{array}{l}\text { ER+ and/or PR+, HER2- and grade III } \\
\text { ER+ and/or PR+, HER2+ }\end{array}$ \\
\hline Her2neu & ER- and PR-, HER2+ \\
\hline $\begin{array}{l}\text { Triple-negative } \\
\text { breast cancer }\end{array}$ & ER- and PR-, HER2- \\
\hline
\end{tabular}

ER-estrogen receptor, $P R$ - progesterone receptor, Her2neu - human epidermal growth factor receptor 2

pathological nodal $(\mathrm{pN})$ stage, margin status, lymphovascular invasion (LVI), endocrine therapy, and chemotherapy. $P$ value $<0.05$ was considered as significant.

\section{Results}

\section{Patient, tumor, and treatment characteristic}

Patient, tumor, and treatment characteristics are summarized in Table 3. Median follow-up was 35 months for all patients (range, 12-71 months). Median age was 60 years (range, 45-78 years). Median pathological tumor (pT) size was $2.1 \mathrm{~cm}$ (range, $0.6-3.2 \mathrm{~cm}$ ). The width of final cut margin varied from 1-17 $\mathrm{mm}$ (median, $8 \mathrm{~mm}$ ). As shown in Table 3, 34.4\% $(n=54)$ of women had luminal A, 36.3\% $(n=57)$ luminal B, 10.8\% $(n=17)$ Her2neu, and $18.5 \%(n=29)$ triple-negative breast cancer (TNBC). As per updated ASTRO consensus, 46 (29.3\%), 106 (67.5\%) and $5(3.2 \%)$ patients belonged to suitable, cautionary, and unsuitable group, respectively. In the cautionary group, most common risk factor was pT size $>2 \mathrm{~cm}(66.0 \%)$, followed by ER negativity $(40.0 \%)$, and LVI positivity $(18.0 \%)$. In the unsuitable group, 2 patients had tumor size $>3 \mathrm{~cm}$, and 3 patients had single nodal metastasis. These were not reported intra-op in frozen section but confirmed on the final histopathology report, which was obtained after the patient had already started on brachytherapy treatment. As per GEC ESTRO consensus for patient's selection, our cohort consisted of $123(78.3 \%), 10(6.4 \%)$, and $24(15.3 \%)$ patients belonging to low, intermediate, and high-risk groups, respectively. Among the high-risk group, most common risk factor was LVI positivity $(87.5 \%)$, and among the intermediate group most common risk factor was age between $40-50$ years $(70 \%)$.

Across the molecular subtype, there was a difference in pT stage $(p=0.04)$ and ASTRO consensus group $(p<0.001)$, as shown in Table 4. Luminal A subtype had a higher percentage $(63 \%)$ of $\mathrm{T} 1$ tumors, as compared to other subtypes. Hence, half of the patients with luminal A subtype belonged to ASTRO suitable category, whereas in luminal B, Her2neu, and TNBC subtypes higher percentage of patients belonged to ASTRO cautionary category (range, 63-100\%) (Table 4). As a result, the use
Table 3. Patient, tumor and treatment characteristic

\begin{tabular}{|c|c|c|}
\hline Characteristic & Group & $N(\%)$ \\
\hline \multirow[t]{3}{*}{ Age group (years) } & $<40$ & 0 \\
\hline & $40-49$ & $7(4.5)$ \\
\hline & $\geq 50$ & $150(95.5)$ \\
\hline \multirow{3}{*}{$\begin{array}{l}\text { Menopausal } \\
\text { status }\end{array}$} & Pre-menopausal & $7(4.5)$ \\
\hline & Post-menopausal & $141(89.7)$ \\
\hline & Peri-menopausal & $9(5.7)$ \\
\hline \multirow[t]{2}{*}{ pT size } & $\leq 2 \mathrm{~cm}$ & $76(48.4)$ \\
\hline & $>2 \mathrm{~cm}$ & $81(51.6)$ \\
\hline \multirow[t]{2}{*}{ pN stage } & Negative (pNo) & $154(98.2)$ \\
\hline & Positive 1-3 (pN1a) & $3(1.8)$ \\
\hline \multirow[t]{2}{*}{ Final margin } & Negative ( $\geq 2 \mathrm{~mm}$ ) & $155(98.7)$ \\
\hline & Close $<2 \mathrm{~mm}$ & $2(1.3)$ \\
\hline \multirow[t]{3}{*}{ Tumor grade } & I & $7(4.5)$ \\
\hline & II & $51(32.5)$ \\
\hline & III & 99 (63.0) \\
\hline \multirow[t]{2}{*}{ EIC } & Positive & $1(0.6)$ \\
\hline & Negative & $156(99.4)$ \\
\hline \multirow[t]{2}{*}{ LVI } & Positive & $21(13.4)$ \\
\hline & Negative & $136(86.6)$ \\
\hline \multirow{2}{*}{$\begin{array}{l}\text { ER (estrogen } \\
\text { receptor) }\end{array}$} & Positive & $111(70.7)$ \\
\hline & Negative & $46(29.3)$ \\
\hline \multirow{2}{*}{$\begin{array}{l}\text { PR (progesterone } \\
\text { receptor) }\end{array}$} & Positive & $96(61.1)$ \\
\hline & Negative & $61(38.9)$ \\
\hline \multirow[t]{2}{*}{ Her2neu status } & Negative & $129(82.2)$ \\
\hline & Positive & $28(17.8)$ \\
\hline \multirow{4}{*}{$\begin{array}{l}\text { Molecular } \\
\text { subtype }\end{array}$} & Luminal A & $54(34.4)$ \\
\hline & Luminal B & $57(36.3)$ \\
\hline & Her2neu & $17(10.8)$ \\
\hline & TNBC & $29(18.5)$ \\
\hline \multirow{3}{*}{$\begin{array}{l}\text { Updated ASTRO } \\
\text { consensus } \\
\text { groups }\end{array}$} & Suitable & $46(29.3)$ \\
\hline & Cautionary & $106(67.5)$ \\
\hline & Unsuitable & $5(3.2)$ \\
\hline \multirow{3}{*}{$\begin{array}{l}\text { GEC-ESTRO risk } \\
\text { groups }\end{array}$} & Low-risk & $123(78.3)$ \\
\hline & Intermediate-risk & $10(6.4)$ \\
\hline & High-risk & $24(15.3)$ \\
\hline \multirow[t]{2}{*}{ Chemotherapy } & Yes & 85 (54.0) \\
\hline & No & $72(46.0)$ \\
\hline \multirow{2}{*}{$\begin{array}{l}\text { Endocrine } \\
\text { therapy }\end{array}$} & Yes & $111(70.7)$ \\
\hline & No & $46(29.3)$ \\
\hline \multirow{2}{*}{$\begin{array}{l}\text { Timing of } \\
\text { implant }\end{array}$} & Intra-operative & $87(55.0)$ \\
\hline & Post-operative & $40(45.0)$ \\
\hline
\end{tabular}

EIC - extensive intraductal component, LVI - lymphovascular invasion, Her2neu - human epidermal growth factor receptor 2, ASTRO - American Society for Therapeutic Radiology and Oncology, GEC-ESTRO - the Groupe Européen de Curietherapie and the European Society for Radiotherapy \& Oncology 
Table 4. Tumor and treatment characteristic across the molecular subtype. Bold numerals = statistically significant, $p<0.05$

\begin{tabular}{|c|c|c|c|c|c|c|}
\hline \multirow[t]{2}{*}{ Characteristics } & \multirow[t]{2}{*}{ Groups } & \multicolumn{4}{|c|}{ Molecular subtype } & \multirow[t]{2}{*}{$p$-value } \\
\hline & & $\begin{array}{l}\text { Luminal A } \\
(n=54)\end{array}$ & $\begin{array}{l}\text { Luminal B } \\
(n=57)\end{array}$ & $\begin{array}{c}\text { Her2neu } \\
(n=17)\end{array}$ & $\begin{array}{c}\text { TNBC } \\
(n=29)\end{array}$ & \\
\hline \multirow[t]{2}{*}{ pT size } & $\leq 2 \mathrm{~cm}$ & $34(63 \%)$ & $25(44 \%)$ & $4(23 \%)$ & $13(46 \%)$ & 0.04 \\
\hline & $>2 \mathrm{~cm}$ & $20(36 \%)$ & $32(66 \%)$ & $13(77 \%)$ & $16(55 \%)$ & \\
\hline \multirow[t]{2}{*}{ p N status } & Negative (pNo) & 53 (98\%) & $56(98 \%)$ & $17(100 \%)$ & $28(96 \%)$ & 0.87 \\
\hline & Positive 1-3 (pN1a) & $1(3 \%)$ & $1(2 \%)$ & 0 & $1(3 \%)$ & \\
\hline \multirow[t]{2}{*}{ LVI } & Positive & $8(16 \%)$ & $10(18 \%)$ & $1(6 \%)$ & $2(7 \%)$ & 0.41 \\
\hline & Negative & $46(84 \%)$ & 47 (82\%) & $16(94 \%)$ & 27 (93\%) & \\
\hline \multirow{3}{*}{$\begin{array}{l}\text { Updated ASTRO } \\
\text { category }\end{array}$} & Suitable & $28(51 \%)$ & $18(31 \%)$ & 0 & $1(3 \%)$ & $<0.001$ \\
\hline & Cautionary & $25(46 \%)$ & $36(63 \%)$ & $17(100 \%)$ & $27(93 \%)$ & \\
\hline & Unsuitable & $1(3 \%)$ & $3(6 \%)$ & 0 & $1(3 \%)$ & \\
\hline \multirow{3}{*}{$\begin{array}{l}\text { Updated ASTRO } \\
\text { category (ER status } \\
\text { replaced by molecular } \\
\text { subtype) }\end{array}$} & Suitable & $28(51 \%)$ & 0 & 0 & 0 & $<0.001$ \\
\hline & Cautionary & $25(46 \%)$ & $54(94 \%)$ & 0 & 0 & \\
\hline & Unsuitable & $1(3 \%)$ & $3(6 \%)$ & $17(100 \%)$ & 29 (100\%) & \\
\hline \multirow{3}{*}{$\begin{array}{l}\text { GEC-ESTRO risk } \\
\text { groups }\end{array}$} & Low-risk & 44 (81\%) & $42(73 \%)$ & $13(76 \%)$ & $24(82 \%)$ & 0.13 \\
\hline & Intermediate-risk & $1(3 \%)$ & $3(6 \%)$ & $3(17 \%)$ & $3(11 \%)$ & \\
\hline & High-risk & $9(16 \%)$ & $12(21 \%)$ & $1(6 \%)$ & $2(7 \%)$ & \\
\hline \multirow[t]{2}{*}{ Chemotherapy } & Yes & $11(21 \%)$ & $35(61 \%)$ & $16(94 \%)$ & $23(79 \%)$ & $<0.001$ \\
\hline & No & $43(79 \%)$ & $22(39 \%)$ & $1(6 \%)$ & $6(21 \%)$ & \\
\hline \multirow[t]{2}{*}{ Endocrine therapy } & Yes & $54(100 \%)$ & 57 (100\%) & 0 & 0 & $<0.001$ \\
\hline & No & 0 & 0 & 17 (100\%) & 29 (100\%) & \\
\hline
\end{tabular}

LVI - lymphovascular invasion, ASTRO - American Society for Therapeutic Radiology and Oncology, ER - estrogen receptor, GEC- ESTRO - the Groupe Européen de Curiethérapie and the European Society for Radiotherapy \& Oncology

of chemotherapy and endocrine therapy varied among the various subtypes. Only nine out of 28 Her2neu positive patients received trastuzumab (one patient received a single dose of trastuzumab peri-operatively as a part of a clinical trial, seven patients received it only for 12 weeks due to financial constraints, and only one patient received full 1 year of trastuzumab). There was no significant difference across subtypes with respect to GEC-ESTRO risk stratification, LVI, and nodal status.

\section{Treatment outcomes}

With a median follow-up of 35 months, a total ten disease failures were documented, which included three isolated local recurrences, one local recurrence with distant metastasis, another with both regional and distant relapse, and 5 patients had only distant failures. Out of the four patients who had ipsilateral breast tumor recurrence (IBTR), one patient had recurrence in the index quadrant, while the other three patients had recurrence in different quadrant of the treated breast. Regional recurrence occurred at the internal mammary chain of lymph nodes. The location of the primary tumor in this patient was in the lower inner quadrant. All the three cases of isolated IBTR were salvaged with modified radical mastectomy and are alive and disease-free till the last follow-up. The other case of IBTR who also developed distant metastasis (liver, brain), received palliative chemotherapy as well as palliative whole brain radiation and was alive with disease till the time of last follow-up. Four patients had died of disease, 2 additional patients died of cardiovascular causes, and one patient died of cerebrovascular cause. Of the 4 deaths related to disease, all had widespread metastatic disease.

\section{Local and locoregional control}

The 3-year actuarial LC, LRC, DMFS, DFS, CSS, and OS as per molecular subtypes, and ASTRO and GEC ESTRO risk categories are summarized in Table 5 . As the ASTRO unsuitable category had only 5 patients, these were analyzed with the cautionary group for all the time to event endpoints to avoid skewed results due to low patient numbers. Four patients developed IBTR, resulting in an overall 3-year actuarial local control rate of $96.5 \%$.

Out of 4 patients who had IBTR, 3 patients belonged to luminal B subgroup and 1 patient belonged to Her2neu 
Table 5. Three-years actuarial outcome by molecular subtype, updated ASTRO category and GEC- ESTRO risk groups. As the number of unsuitable cases ASTRO Consensus was only five, unsuitable and cautionary analyzed as one category for statistical purpose. Clinical outcomes that were studied included local control (LC), locoregional control (LRC), distant metastasis-free survival (DMFS), disease-free survival (DFS), cause specific survival (CSS), and overall survival (OS). All the time to event data were calculated from the date of surgery. Bold numerals show statistically significant data, $p<0.05$

\begin{tabular}{|c|c|c|c|c|c|c|c|c|c|c|c|c|c|}
\hline & & $\begin{array}{l}\mathrm{LC} \\
(\%) \\
\end{array}$ & $p$-value & $\begin{array}{l}\text { LRC } \\
(\%) \\
\end{array}$ & $p$-value & $\begin{array}{c}\text { DMFS } \\
(\%)\end{array}$ & $p$-value & $\begin{array}{l}\text { DFS } \\
(\%)\end{array}$ & $p$-value & $\begin{array}{l}\text { CSS } \\
(\%)\end{array}$ & $p$-value & $\begin{array}{l}\text { OS } \\
(\%)\end{array}$ & $p$-value \\
\hline Overall & & 96.5 & & 96.5 & & 95.6 & & 93.1 & & 98.3 & & 97.6 & \\
\hline \multirow{4}{*}{$\begin{array}{l}\text { Molecular } \\
\text { subtype }\end{array}$} & Luminal A & 100 & \multirow[t]{4}{*}{0.19} & 100 & \multirow[t]{4}{*}{0.41} & 100 & \multirow[t]{4}{*}{0.01} & 100 & \multirow[t]{4}{*}{0.007} & 100 & \multirow[t]{4}{*}{0.006} & 98.1 & \multirow[t]{4}{*}{0.05} \\
\hline & Luminal B & 93.3 & & 93.3 & & 97.6 & & 93.3 & & 100 & & 100 & \\
\hline & Her2neu & 87.5 & & 87.5 & & 71.6 & & 61.4 & & 82.0 & & 82.0 & \\
\hline & TNBC & 100 & & 96.4 & & 96.4 & & 96.4 & & 92.9 & & 92.9 & \\
\hline \multirow{2}{*}{$\begin{array}{l}\text { Updated } \\
\text { ASTRO } \\
\text { category }\end{array}$} & Suitable & 97.1 & \multirow[t]{2}{*}{0.80} & 97.1 & \multirow[t]{2}{*}{0.61} & 100 & \multirow[t]{2}{*}{0.27} & 97.1 & \multirow[t]{2}{*}{0.38} & 100 & \multirow[t]{2}{*}{0.13} & 100 & \multirow[t]{2}{*}{0.21} \\
\hline & Cautionary & 96.1 & & 95.1 & & 93.5 & & 91.0 & & 97.4 & & 96.5 & \\
\hline \multirow{3}{*}{$\begin{array}{l}\text { GEC-ESTRO } \\
\text { risk group }\end{array}$} & Low & 96.6 & \multirow[t]{3}{*}{0.56} & 96.6 & \multirow[t]{3}{*}{0.42} & 97.5 & \multirow[t]{3}{*}{0.20} & 94.4 & \multirow[t]{3}{*}{0.49} & 99.2 & \multirow[t]{3}{*}{0.007} & 98.4 & \multirow[t]{3}{*}{0.11} \\
\hline & $\begin{array}{l}\text { Inter- } \\
\text { mediate }\end{array}$ & 100 & & 90.0 & & 87 & & 78.8 & & 85.7 & & 85.7 & \\
\hline & High & 94.4 & & 94.4 & & 100 & & 94.4 & & 100 & & 100 & \\
\hline
\end{tabular}

ASTRO - American Society for Therapeutic Radiology and Oncology, GEC-ESTRO - the Groupe Européen de Curiethérapie and the European Society for Radiotherapy \& Oncology

subgroup. The luminal B subgroup patients who failed locally, when analyzed according to Her2neu status, 2 cases were ER+/Her2neu-/grade III, and the other case was ER+/Her2neu+/grade III (this case had local recurrence in index quadrant). The true in-field recurrence pa-

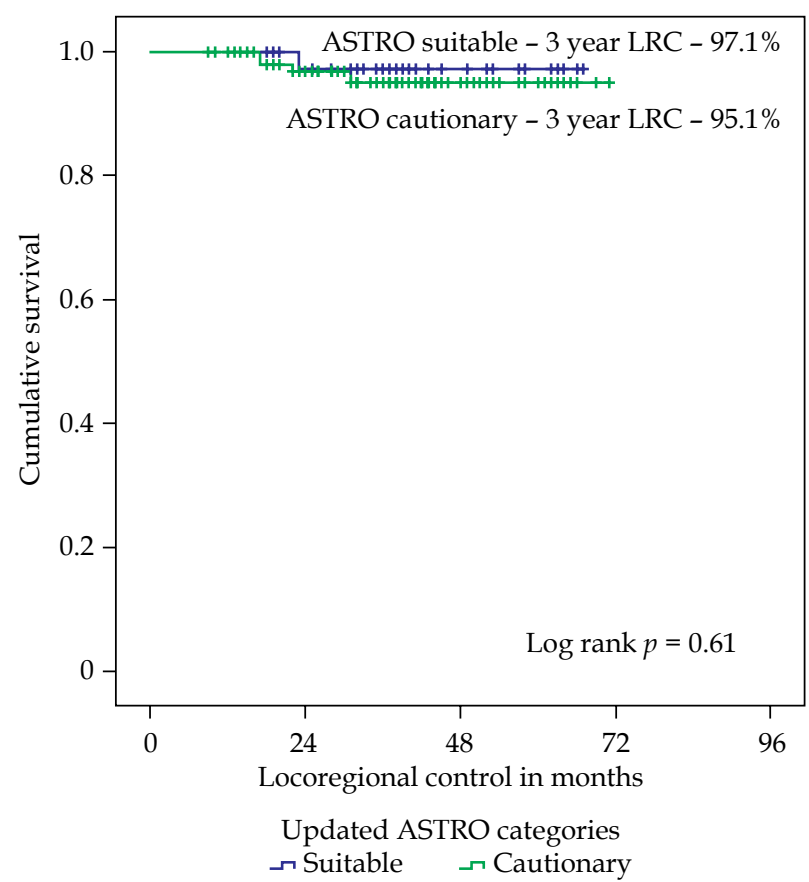

Fig. 1. Locoregional control (LRC) by updated ASTRO categories (unsuitable and cautionary analyzed as one category) tient and Her2neu subgroup patient did not receive trastuzumab due to financial constrain. No IBTR occurred in patients with TNBC subtype. There was one regional nodal failure (RNF), which occurred in the TNBC subtype with actuarial regional nodal recurrence (RNR) rate of $0.7 \%$ at 3 years.

One isolated IBTR occurred in the ASTRO suitable/ GEC-ESTRO low-risk group, and the other occurred in ASTRO unsuitable/GEC-ESTRO high-risk group (unsuitable $/$ high-risk in view of $\mathrm{pT}$ size $=3.1 \mathrm{~cm}$ ). The other two IBTR occurred in ASTRO cautionary/GEC-ESTRO lowrisk group (cautionary in view of T size $>2 \mathrm{~cm}$ and ER receptor status negative). The 3 -year actuarial IBTR was $2.9 \%, 3 \%$, and $25 \%$ in ASTRO suitable, cautionary, and unsuitable group, respectively, and $3.4 \%, 0 \%$, and $5.6 \%$ in GEC-ESTRO low, intermediate, and high-risk group, respectively. The single RNF occurred in the ASTRO cautionary group (in view of ER negativity and final cut margin $<2 \mathrm{~mm}$ )/GEC-ESTRO intermediate risk group (in view of close margin). The survival curves for locoregional control as per updated ASTRO categories, molecular subtype, and GEC-ESTRO risk groups are shown in Figures 1-3, respectively. Univariate analysis demonstrated that neither molecular subtype nor the APBI selection criteria (update ASTRO/ESTRO) were predictive for local or locoregional outcome. The patient data set was re-analyzed for LC and LRC as per updated ASTRO category by replacing ER status by molecular subtype to find out whether inclusion of molecular subtype in ASTRO risk stratification has important impact on local outcomes. For purpose of this analysis, luminal A subtype alone in absence of other risk factors was considered as suitable cat- 


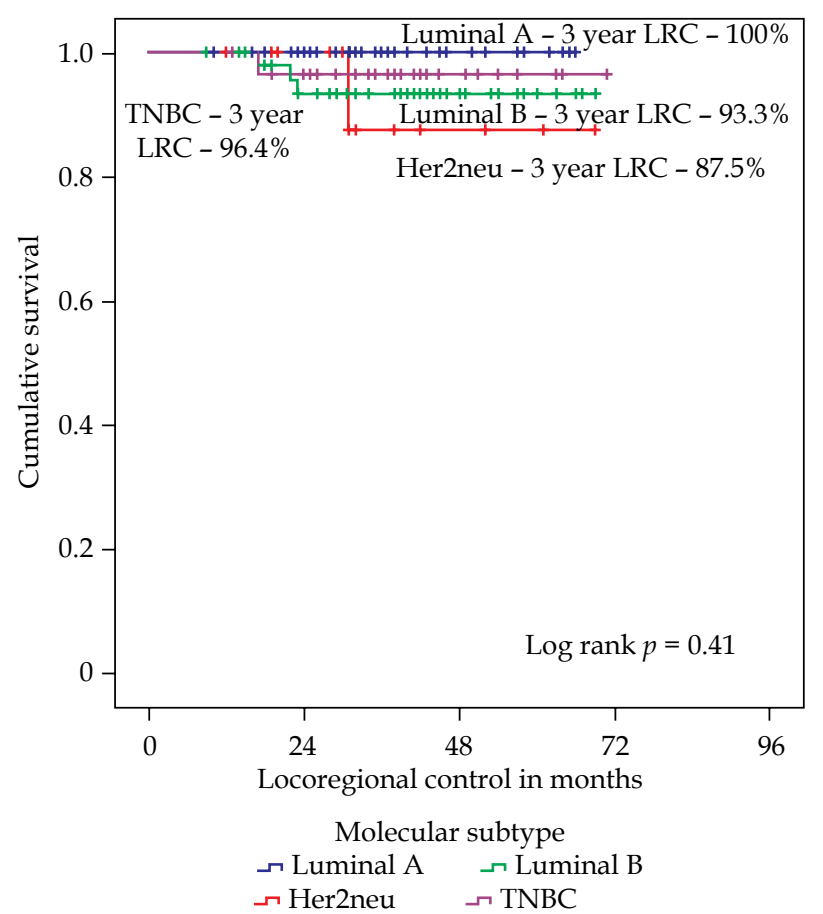

Fig. 2. Actuarial locoregional control (LRC) by molecular subtype

egory, luminal B subtype was considered as cautionary category (provided any other unsuitable risk factor was not present), and Her2neu and TNBC was considered as unsuitable category. As per this analysis, no statistical significant difference in LC and LRC was found across the ASTRO categories ( $p=0.22$ for LC, and $p=0.28$ for $\mathrm{LRC}$ ). Other variables including age, $\mathrm{pT}$ size, $\mathrm{pN}$ stage, margin status, estrogen receptor (ER), progesterone receptor (PR), Her2neu status, lymphovascular invasion (LVI), extensive intraductal component (EIC), grade, GEC-ESTRO stratification, receipt of chemotherapy, and hormonal therapy were not associated with a significantly increased risk of local or locoregional recurrence.

Distant metastasis, disease-free survival, cause specific survival, $O S$

Seven patients had distant metastasis, resulting in overall 3-year actuarial rates of $4.4 \%$. The 3 -year actuarial distant recurrence rates by subtype were $0 \%$ for luminal A, $2.4 \%$ for luminal B $(n=2), 28.4 \%$ for Her2 $(n=3)$, and $3.6 \%$ for TNBC $(n=2)$. Her2neu subtype was significantly associated with higher risk of distant metastasis than luminal A $(p=0.002)$ and luminal B $(p=0.029)$ subtypes. Only 1 out of 3 patients in Her2 group who failed in distant site had earlier received single dose of peri-operative herceptin. TNBC subtype did not demonstrate statistically significant difference in DFS over luminal A $(p=0.08)$, luminal B $(p=0.97)$, and Her2neu $(p=0.06)$ subtypes. This translated into significantly lower DFS, CSS, and OS in the Her2 subtype than the luminal subtypes. Out of the seven patients who had distant recurrences, six belonged to ASTRO cautionary group and one belonged to ASTRO suitable group. When analyzed pairwise over stratum,

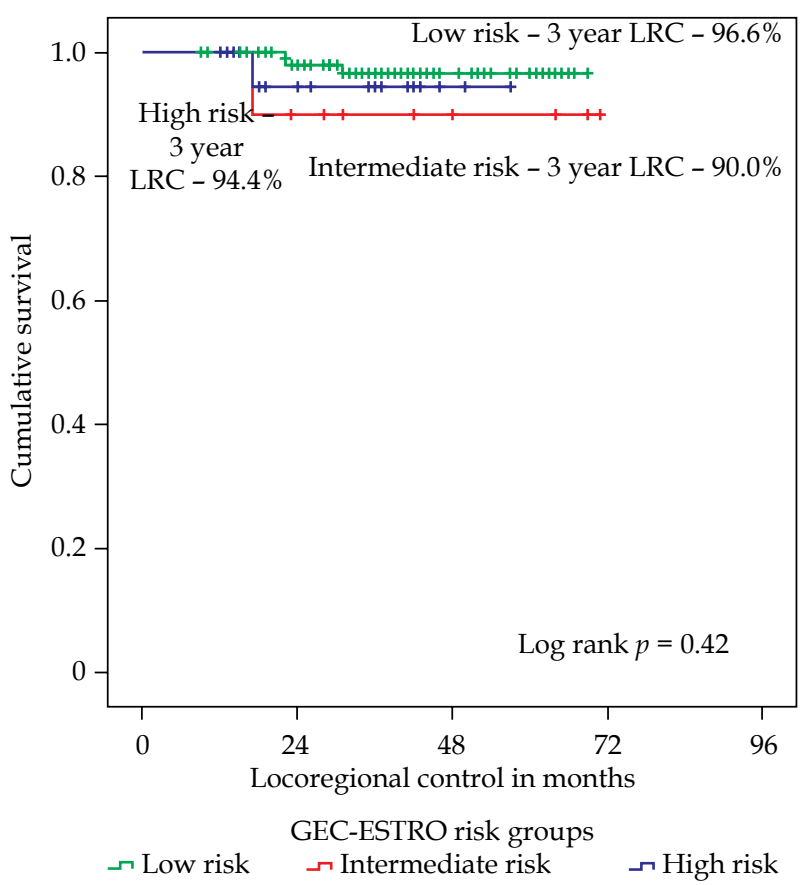

Fig. 3. Actuarial locoregional control (LRC) by GEC-ESTRO risk category

intermediate risk group was also associated with significantly lower CSS $(p=0.03)$. ER status $(p=0.03)$, PR status $(p=0.03)$, and final margin status $(<2 \mathrm{~mm})(p<0.001)$ were the other variables which demonstrated significant difference in DFS in univariate analysis, whereas age group, use of chemotherapy, and endocrine therapy were not associated with significant difference in DFS.

\section{Discussion}

Over the last two decades, APBI has emerged as an appealing and effective alternative to WBI for selected early stage women. Results from two randomized trials have shown that MIB-APBI has similar local control rate compared to WBI $[1,20]$. These two trials have used multicatheter interstitial brachytherapy for APBI. Ten-year results of the Budapest randomized trial demonstrated that after a median follow-up of 10.2 years, the 10-year actuarial rate of LR was 5.9\% and 5.1\% in PBI and WBI arms, respectively $(p=0.77)$. Neither there was a significant difference in the 10 -year probability of OS (80\% vs. $82 \%)$, CSS (94\% vs. $92 \%$ ), and DFS (85\% vs. $84 \%$ ). Significantly better cosmetic outcome was achieved with interstitial implants [20]. Similarly, in the recently published GEC-ESTRO trial, the 5-year cumulative incidence of local recurrence was $1.44 \%$ with APBI and $0.92 \%$ with whole-breast irradiation $(p=0.42)$ [1]. We have reported earlier a case-control study comparing disease control, cosmesis, and complications in patients with early breast cancer undergoing MIB-APBI versus WBI. At the median follow-up of 43.05 months in APBI and 51.08 months in WBRT, there was no difference in overall survival (OS), disease-free survival (DFS), late arm edema, and 
symptomatic fat necrosis between the two groups [21]. Cost effectiveness of MIB-APBI technique was shown in a study by Harat et al. [2]. This study has analyzed average cost of treatment and incremental cost-effectiveness ratio of MIB-APBI compared to WBI in early breast cancer patients over a 5-year period, based on the results of GEC-ESTRO trial. The average cost of treatment with APBI was lower than for WBI, and the study concluded that the use of APBI as an alternative to WBI would substantially reduce healthcare expenditures in both 2013 and 2025, assuming even an increase in the price per single APBI procedure [2]. Cosmetic outcome is an important endpoint in early breast cancer trials. Bitter et al. have shown that APBI treated patients have higher cosmetic satisfaction than patients treated with WBRT [3]. Excellent cosmetic outcomes with acceptable toxicity profile was reported by Akhtari et al. in their APBI series using single-entry multi-lumen/catheter applicators with skin spacing of $\leq 7 \mathrm{~mm}$ [22].

Proper patient selection carries an important implication on outcomes of APBI. The chief rationale behind patient selection for APBI is by defining favorable clinical and pathological criteria for minimal risk for local tumor recurrence. Though several consensus groups have recommended the use of guidelines for patient selection, most of these guidelines are based on systematic review of the APBI literature and expert opinion, rather than on individual patient data from APBI series that can identify subset of patients with higher local recurrence rates when treated with APBI.

In 2009, the ASTRO research committee and subsequently in 2010, GEC-ESTRO consensus panel published consensus guidelines based on published evidence complemented by expert opinion. In the last few years, several publications have analyzed the validity of original ASTRO and GEC-ESTRO consensus groups in predicting clinical outcome $[23,24,25,26,27,28]$. In a study by Vicini et al., though ASTRO suitable category predicted for a low-risk of IBTR in patients treated with MIB-APBI, there were no statistically significant differences in the IBTR ( $2.6 \%$ vs. $7.8 \%$ vs. 2.5 , respectively, $p=0.85)$ and RNF rates among the three ASTRO categories [23]. However, the rate of distant metastasis (DM) was significantly higher in cautionary and unsuitable categories $(7.1 \%$ and $11.2 \%$, respectively) than in suitable category $(0 \%$, $p=0.01$ ). Similar study by Mc Haffey et al., in which $93 \%$ patients were treated with MIB-APBI, the 5-year actuarial incidence of IBTR were $1.6 \%, 4.8 \%$, and $6.6 \%$, and the 5 -year locoregional recurrence rates were $1.6 \%, 4.8 \%$, and $8.7 \%$ in suitable, cautionary, and unsuitable categories, respectively [24]. This study, along with similar other studies, showed that "cautionary" category patients have acceptably low rates of IBTR $[25,26]$. Shaitelman et al. have analyzed the clinical outcome based on ASTRO consensus in 1,449 APBI patients treated under MammoSite Registry Trial [27]. At a median follow-up of 53.5 months, the 5-year actuarial rates of IBTR for the suitable, cautionary, and unsuitable categories were $2.59 \%, 5.43 \%$, and $5.28 \%$, respectively $(p=0.18)$. Only negative estrogen receptor status was associated with increased risk of IBTR. No difference in DFS, CSS, and OS was found among the groups, but DM rate differed significantly $(0.87 \%, 4.44 \%$, and $3.01 \%$, respectively, $p=0.03$ ). Similar results were obtained in a pooled analysis from William Beaumont Hospital (WBH) $(n=678$; interstitial, $n=221$; balloon, $n=255$; 3D-CRT, $n=206$ ) and the American Society of Breast Surgeons (ASBrS) MammoSite Registry Trial $(n=1449)$, and the authors concluded that the ASTRO CS guidelines did not adequately differentiate patients in an increased risk of IBTR or tumor bed failure in this large patient cohort [28].

Although, several studies have reported outcomes based on original ASTRO CS, only one study has shown statistically different rates of IBTR between risk groups [29]. None of the brachytherapy series reported significantly worst IBTR rates as per ASTRO CS groups. The study by Leonardi et al. included 1,822 patients treated with intra-operative electrons (ELIOT) off protocol, and showed significant difference in 5 years IBTR throughout all the 3 groups, when categorized according to ASTRO guideline $(1.5 \%, 4.4 \%$, and $8.8 \%$, respectively; $p=0.0003$ ) [29]. Although there was no difference in regional node relapse rate, the rate of distant metastases was significantly different in the unsuitable group compared with the other two groups, having a significant impact on PFS, CSS, and OS. Factors associated with increased risk of local relapse were age $<50$ years, tumor size $>2 \mathrm{~cm}$, high grade, diffuse LVI, estrogen receptor negative status, multicentricity, and positive lymph nodes. The application of GEC-ESTRO recommendations in the same cohort of patients resulted in statistically significant differences in all the clinical outcomes (except RNF rate) among the risk groups [10]. The incidence of IBTR was similar in intermediate risk and in high-risk groups (7.4\% and $7.7 \%$, respectively), and was significantly higher than in low-risk group patients $(1.9 \%, p=0.001)$. Estrogen receptor status and high-grade tumors were identified as risk factors for the development of IBTR. However, both these risk factors are not included in the GEC-ESTRO recommendation. Aliyev et al. have reported early results of two cohorts of patients with GEC-ESTRO low and intermediate risk of early breast cancer treated with APBI, using different schedules of multicatheter brachytherapy 32 Gy/8 fractions/4 days and 35 Gy/7 fractions/4 days [30]. After a median follow-up of 3 years, they did not find any difference between the two fractionation schedules in terms of disease-free survival and early and late toxicity of the treatment, concluding that an increase of fraction size did not lead to higher probability of fat necrosis. According to their findings, APBI was also feasible for intermediate-risk early breast cancer patients. In our institution, we had been using the APBI fractionation regimen of $34 \mathrm{~Gy} / 10$ fractions/ 5 days as per ABS recommendation [6], but after the publication of the GEC-ESTRO trial in 2016, we have changed the fractionation schedules to $32 \mathrm{~Gy} / 8$ fractions/ 4 days.

In all the studies, which are discussed here, stratification of patients was based on the original ASTRO CS, which was published in 2009. Recently, ASTRO guidelines have been revised and updated [9]. The new recommendations include lowering the age in the suitable group from 60 to 50 years and in the cautionary group 
to 40 years for patients who meet all other elements for suitable. Hence, now the age cut off is similar to the GECESTRO risk groups. Shah et al. have reviewed the available patient selection guidelines for brachytherapy-based APBI, and were of the opinion that the updated ASTRO consensus statement has incorporated much of the recent APBI data and provides a simpler set of guidelines for clinicians [31]. In the current study, we have reported the outcome as per both the updated ASTRO and ESTRO guidelines. To the best of our knowledge, this is the first report on such kind of analysis.

There is growing evidence that molecular phenotype has prognostic significance for locoregional recurrence risk both following APBI and WBI. In a study by Nguyen et al., both the HER-2 subtype (adjusted hazard ratio [AHR] 9.2; 95\% CI: 1.6-51, $p<0.012$ ) and the basal subtype (AHR 7.1; 95\% CI: 1.6-31, $p<0.009)$ were associated with an increased risk of local recurrence [11]. In another study by Voduc et al., HER-2-enriched and basal subtypes demonstrated an increased risk of regional recurrence for patients undergoing BCS, while luminal B, luminal-HER2, HER2-enriched, and basal subtypes were all associated with an increased risk of local and regional relapse after mastectomy [12]. These studies were criticized for higher local and regional recurrence rate attributed to the underuse of systemic therapy, including the absence of adjuvant trastuzumab. Albert et al. limited their analysis to tumors $<1 \mathrm{~cm}$, which were less likely to be treated with chemotherapy [13]. ER/ PR-negative and HER-2-positive status were shown to be independently predicted for locoregional recurrence (LRR). Several studies have shown that the basal or TNBC subtypes are associated with an increased risk of both LR and distant metastases [11,14].

The literature on APBI and molecular subtype is conflicting. Wilkinson et al. showed similar 5-year actuarial IBTR, RNF, DM, and OS in the TNBC patients compared to receptor positive patients who were treated with APBI [15]. In a recently published study by the same author, the 5-year local control rates after APBI and systemic therapy were similar for luminal, HER-2, and basal phenotypes of early-stage breast cancer [16]. In this study, only 2 out of $5(40 \%)$ Her2neu subtype patients received trastuzumab. In contrast, Pashtan et al. reported on a series of 99 stage I patients treated with $3 \mathrm{D}$ conformal external beam APBI. Three of the six LR occurred in triple negative (TN) patients, yielding a LR of $32.5 \%$ among TN patients, compared to only $3 \%$ among the other subtypes $(p=0.0001)$ [17]. All patients who developed LR had received systemic treatment. On multivariate analysis, triple-negative phenotype was the only predictor of LR, with borderline statistical significance ( $p=0.052, \log$-rank test) after adjusting for tumor grade. The study also analyzed the possible effect of the surgery-to radiation therapy interval on the risk of IBTR. Two of the 3 TNBC patients with IBTR had chemotherapy prior to RT; RT commenced on days 62, 105, and 174 after lumpectomy (mean, 114 days). The authors discussed that mature follow-up and delayed initiation of radiotherapy could have accounted for earlier development of IBTR in TN patients. On the contrary, as majority of patients (55\%) underwent intra-operative procedure and early institution of radiation, overall very few recurrences occurred in our cohort. Only in Her2neu subgroup relatively lesser number of patients underwent intra-operative implant compared to other subgroups $(41 \%, 48 \%, 54 \%, 63 \%$ in Her2neu, TNBC, luminal B, and luminal A subgroup, respectively). Alderson et al. conducted a study with 582 breast cancers patients treated with MIB-APBI [18]. With a median follow-up time of 5.4 years, the 5 -year IBTR rate was $4.7 \%$ overall, $3.5 \%$ for luminal A, 4.1\% for luminal B, 5.2\% for luminal Her2, $13.3 \%$ for Her2, and $11.3 \%$ for triple-negative breast cancer. Positive surgical margins and high grade were associated with increased risk for IBTR, as was Her2 subtype in comparison with luminal A subtype. RNF risk was higher for women with Her2 subtype compared to the other four subtypes and for luminal B compared to luminal A subtype. The clinical outcome of our cohort is comparable to that reported by Alderson et al. Orecchia et al. have reviewed the available literature on the role of molecular subtype in predicting local recurrence in patients treated with APBI. They concluded that there is growing evidence in literature that molecular subtypes can have a great impact also in locoregional management of breast cancer but needs to be validated in datasets [32].

Results from the above mention studies shows that both molecular subtype and the risk grouping based on ASTRO and GEC-ESTRO guideline may have impact on LC and LRC of early breast cancer patient treated with APBI. Our study is the first study to report clinical outcome based on both molecular subtype and updated ASTRO and GEC-ESTRO risk categories to determine the need for inclusion of molecular subtype in the selection criteria for APBI, though the follow-up is short. In our study, none of the guidelines (updated ASTRO and ESTRO) predicted risk of local or locoregional recurrence, though the unsuitable category was analyzed with cautionary category owing to few number of cases in unsuitable category. However, there was no statistical significant difference between the 3-year actuarial LC and LRC rates among the molecular subtypes, or according to ER, PR, Her2neu status, three cases of IBTR occurred in luminal $B$ subtype. This can be possibly explained by the fact that luminal B subtype had a higher percentage of T2 tumors, as compared to luminal A or TNBC subtypes. We did not observe a statistically significant increased risk of local recurrence within TNBC subgroup. These findings are in agreement to the study results reported by Wilkinson et al., in which IBTR rates at 5 years did not differ significantly across the molecular subgroups; 5 out of 6 cases of IBTR occurred in luminal subtype and the TNBC subtype was not associated with higher risk of local recurrence [15]. Evidence has shown that use of trastuzumab may lower the LR rates for the HER2+ subtypes [33]. In our series, only 1 out of 28 Her2neu positive patients received trastuzumab for 1 year and others were unable to receive due to financial constraints. Moreover, the two patients who developed IBTR and also had Her2neu positive disease, were unable to receive trastuzumab. This could be a possible explanation for lower LC rate. The significantly higher rate of distant metastasis in the Her2 subtype may also be explained due to lack of targeted therapy. This also indicates that HER2+ women not receiving trastuzum- 
ab should be cautiously selected for APBI. As a result, prospective patients with HER2+ disease are not being considered for APBI at our institute if not receiving trastuzumab. GEC-ESTRO risk grouping did not predict for LC and LRC in this cohort of patients at relatively short follow-up, but the intermediate risk group was associated with higher rates of DM translating into lower CSS than the low-risk group. Limitations of the current analysis include its retrospective nature, small absolute number of local failures with a relatively small sample size, and limited length of follow-up. Tumor grade was taken as a surrogate measure of proliferation, and approximation of molecular subtype was done accordingly. Relatively lesser number of patients received trastuzumab in Her2+ group, due to financial constraints.

\section{Conclusions}

None of the APBI guidelines (updated ASTRO and ESTRO) predicted risk of local or locoregional recurrence in our study population. Though local or locoregional outcome was not significantly different across the molecular subtypes in this cohort of patients with relatively short follow up, DMFS, DFS, CSS, and OS were significantly lower with Her2neu subtype. As molecular subtyping can be easily done, it will provide added value to conventional risk factors in selecting patients for APBI. However, additional follow-up will be needed to recommend inclusion of molecular subtype (or at least HER2 receptor status) in patient's selection criteria for APBI.

\section{Disclosure}

Authors report no conflict of interest.

\section{References}

1. Strnad V, Ott OJ, Hildebrandt G et al. 5-year results of accelerated partial breast irradiation using sole interstitial multicatheter brachytherapy versus whole-breast irradiation with boost after breast-conserving surgery for low-risk invasive and in-situ carcinoma of the female breast: a randomised, phase 3, non-inferiority trial. Lancet 2016; 387: 229-238.

2. Harat A, Harat M, Makarewicz R. Whole breast irradiation vs. APBI using multicatheter brachytherapy in early breast cancer-simulation of treatment costs based on phase 3 trial data. J Contemp Brachytherapy 2016; 8: 505-511.

3. Bitter SM, Heffron-Cartwright P, Wennerstrom et al. WBRT vs. APBI: an interim report of patient satisfaction and outcomes. J Contemp Brachytherapy 2016; 8: 17-22.

4. Smith BD, Arthur DW, Buchholz TA et al. Accelerated partial breast irradiation consensus statement from the American Society for Radiation Oncology (ASTRO). Int J Radiat Oncol Biol Phys 2009; 74: 987-1001.

5. Polgár C, Van Limbergen E, Pötter R et al. Patient selection for accelerated partial-breast irradiation (APBI) after breast-conserving surgery: recommendations of the Groupe Europeen de Curietherapie-European Society for Therapeutic Radiology and Oncology (GEC-ESTRO) breast cancer working group based on clinical evidence (2009). Radiother Oncol 2010; 94: 264-273.

6. Shah C, Vicini F, Wazer DE et al. The American Brachytherapy Society consensus statement for accelerated partial breast irradiation. Brachytherapy 2013; 12: 267-277.
7. The American Society of Breast Surgeons: Consensus Statement for Accelerated Partial Breast Irradiation. Available at: https://www.breastsurgeons.org/new_layout/about/ statements/PDF_Statements/APBI.pdf; Revised August 15, 2011. Accessed: 20 August 2017.

8. Radiation Therapy Oncology Group. RTOG 0413/NSABP B-39 Study Protocol. Available at: http://www.rtog.org/members/protocols/0413/0413.pdf. Accessed: January 8, 2018.

9. Correa C, Harris EE, Leonardi MC et al. Accelerated partial breast irradiation: executive summary for the update of an ASTRO evidence-based consensus statement. Pract Radiat Oncol 2017; 7: 73-79.

10. Leonardi MC, Maisonneuve P, Mastropasqua MG et al. Accelerated partial breast irradiation with intraoperative electrons: Using GEC-ESTRO recommendations as guidance for patient selection. Radiother Oncol 2013; 106: 21-27.

11. Nguyen PL, Taghian AG, Katz MS et al. Breast cancer subtype approximated by estrogen receptor, progesterone receptor, and HER-2 is associated with local and distant recurrence after breast-conserving therapy. J Clin Oncol 2008; 26: 2373-2378.

12. Voduc KD, Cheang MC, Tyldesley $S$ et al. Breast cancer subtypes and the risk of local and regional relapse. J Clin Oncol 2010; 28: 1684-1691.

13. Albert JM, Gonzalez-Angulo AM, Guray M et al. Estrogen/ progesterone receptor negativity and HER2 positivity predict locoregional recurrence in patients with T1a, bN0 breast cancer. Int J Radiat Oncol Biol Phys 2010; 77: 1296-1302.

14. Banerjee S, Reis-Filho JS, Ashley S et al. Basal-like breast carcinomas: clinical outcome and response to chemotherapy. J Clin Pathol 2006; 59: 729-735.

15. Wilkinson JB, Reid RE, Shaitelman SF et al. Outcomes of breast cancer patients with triple negative receptor status treated with accelerated partial breast irradiation. Int J Radiat Oncol Biol Phys 2011; 81: e159-164.

16. Wilkinson JB, Shah C, Amin M et al. Outcomes According to Breast Cancer Subtype in Patients Treated with Accelerated Partial Breast Irradiation. Clin Breast Cancer 2017; 17: 55-60.

17. Pashtan IM, Recht A, Ancukiewicz M et al. External beam accelerated partial-breast irradiation using 32 Gy in 8 twice-daily fractions: 5-year results of a prospective study. Int J Radiat Oncol Biol Phys 2012; 84: e271-277.

18. Anderson BM, Kamrava M, Wang PC et al. Locoregional recurrence by molecular subtype after multicatheter interstitial accelerated partial breast irradiation: Results from the Pooled Registry of Multicatheter Interstitial Sites research group. Brachytherapy 2016; 15: 788-795.

19. Gurram L, Wadasadawala T, Joshi K et al. Multi-catheter interstitial brachytherapy for partial breast irradiation: an audit of implant quality based on dosimetric evaluation comparing intra-operative versus post-operative placement. J Contemp Brachytherapy 2016; 8: 116-121.

20. Polgár C, Fodor J, Major T et al. Breast-conserving therapy with partial or whole breast irradiation: ten-year results of the Budapest randomized trial. Radiother Oncol 2013; 108: 197-202.

21. Wadasadawala T, Sarin R, Budrukkar A et al. Accelerated partial-breast irradiation vs conventional whole-breast radiotherapy in early breast cancer: A case-control study of disease control, cosmesis, and complications. J Cancer Res Ther 2009; 5: 93-101.

22. Akhtari M, Abboud M, Szeja S et al. Clinical outcomes, toxicity, and cosmesis in breast cancer patients with close skin spacing treated with accelerated partial breast irradiation (APBI) using multi-lumen/catheter applicators. J Contemp Brachytherapy 2016; 8: 497-504.

23. Vicini F, Arthur D, Wazer D et al. Limitations of the American Society of Therapeutic Radiology and Oncology Consen- 
sus Panel guidelines on the use of accelerated partial breast irradiation. Int J Radiat Oncol Biol Phys 2011; 79: 977-984.

24. McHaffie DR, Patel RR, Adkison JB et al. Outcomes after accelerated partial breast irradiation in patients with ASTRO consensus statement cautionary features. Int J Radiat Oncol Biol Phys 2011; 81: 46-51.

25. Stull TS, Goodwin MC, Gracely EJ et al. A single-institution review of accelerated partial breast irradiation in patients considered "cautionary" by the American Society for Radiation Oncology. Ann Surg Oncol 2012; 19: 553-559.

26. Christoudias MK, Collett AE, Stull TS et al. Are the American Society for Radiation Oncology guidelines accurate predictors of recurrence in early stage breast cancer patients treated with balloon-based brachytherapy? Intern J Surg Oncol 2013; 829050.

27. Shaitelman SF, Vicini FA, Beitsch P et al. Five-year outcome of patients classified using the American Society for Radiation Oncology consensus statement guidelines for the application of accelerated partial breast irradiation. Cancer 2010; 116: 4677-4685.

28. Wilkinson JB, Beitsch PD, Shah C et al. Evaluation of current consensus statement recommendations for accelerat ed partial breast irradiation: a pooled analysis of William Beaumont Hospital and American Society of Breast Surgeon MammoSite Registry Trial Data. Int J Radiat Oncol Biol Phys 2012; 85: 1179-1185.

29. Leonardi MC, Maisonneuve P, Mastropasqua MG et al. How do the ASTRO consensus statement guidelines for the application of accelerated partial breast irradiation fit intraoperative radiotherapy? A retrospective analysis of patients treated at the European Institute of Oncology. Int J Radiat Oncol Biol Phys 2012; 83: 806-813.

30. Aliyev JA, Isayev IH, Akbarov KS et al. High-dose-rate interstitial brachytherapy for accelerated partial breast irradiation-trial results of Azerbaijan National Center of Oncology. J Contemp Brachytherapy 2017; 9: 106-111.

31. Shah C, Wobb J, Manyam B et al. Accelerated partial breast irradiation utilizing brachytherapy: patient selection and workflow. J Contemp Brachytherapy 2016; 8: 90-94.

32. Orecchia R, Leonardi MC. Partial breast irradiation: targeting volume or breast molecular subtypes? Breast 2013; 22 Suppl 2: S137-140.

33. Billar JA, Dueck AC, Stucky CC et al. Triple-negative breast cancers: unique clinical presentations and outcomes. Ann Surg Oncol 2010; 17: 384-390. 